\title{
Corrente Russa associada ao ultrassom ou a fonoforese reduz o fibro edema gelóide
}

\section{Russian Current associated with ultrasound or phonophoresis reduces cellulite}

\author{
Anna Y. M. Rodrigues ${ }^{1}$; Jeanine Rolin¹; Mariana V. de Sáli Layna H. C. Silveiral; Morgana \\ Duarte da Silva, ${ }^{1,2}$ \\ 1 Departamento de Fisioterapia - Universidade Federal do Pampa - Unipampa. Uruguaiana, RS - Brasil. \\ 2 Programa Multicêntrico de Pós-graduação em Ciências Fisiológicas, Universidade Federal do Pampa - Unipampa. Uruguaiana, RS - \\ Brasil. \\ Endereço para correspondência: \\ Morgana Duarte da Silva \\ Universidade Federal do Pampa - Unipampa - Campus Uruguaiana \\ Logradouro: BR 472 - Km 592 - Prédio 700 \\ 97500-970 - Uruguaiana - RS [Brasil] \\ dasilvamdbrazil@gmail.com
}

\begin{abstract}
Resumo
Introdução: O fibro edema gelóide (FEG) ocasiona deformações locais no tecido subcutâneo. Correntes excito motoras, como a corrente russa (CR), e métodos que associam efeitos mecânicos e térmicos no tecido, como o ultrassom (US), são usados para tratar a FEG. O US pode ainda ser aplicado com a fonoforese (FN) para aumentar a absorção percutânea de princípios ativos. Objetivo: Verificar o efeito da CR associada ao US ou à FN no tratamento da FEG em glúteos e coxas de mulheres jovens. Métodos: Participaram mulheres com FEG que realizaram 10 sessões de CR com US ou com FN. As voluntárias foram avaliadas antes e após os tratamentos. Resultados: Obteve-se diminuição da espessura das dobras cutâneas, redução do grau de FEG, redução da distorção da imagem corporal e índice de satisfação elevado das voluntárias com os resultados de ambos tratamentos, porém a FN associada à CR apresentou efeito mais evidente. Conclusão: Os tratamentos utilizados fornecem resultados rápidos e satisfatórios na redução do FEG.
\end{abstract}

Descritores: Celulite; Imagem Corporal; Terapia de Estimulação Elétrica; Terapia Ultrassônica; Fonoforese.

\footnotetext{
Abstract

Introduction: Geloid fibroedema (GF) or Cellulitis causes local deformations in the subcutaneous tissue. Electric Stimulation Therapy, such as the Russian Current (RC), and methods associating mechanical and thermal effects on tissue, such as Ultrasound (US), are used to treat GF. US can still be applied with Phonophoresis $(\mathrm{Ph})$ to increase percutaneous absorption of active principles. Objective: To verify the effect of RC associated with US or Ph in the treatment of GF in glutes and thighs of young women. Methods: GF women who performed 10 sessions of RC with US or with Ph participated. The volunteers were evaluated before and after the treatments. Results: There was a reduction in the thickness of the skin folds, reduction of the GF degree, reduction of the body image distortion and high index of satisfaction of the volunteers with the results of both treatments, but the $\mathrm{Ph}$ associated to the $\mathrm{CR}$ showed a more evident effect. Conclusion: The treatments used provide fast and satisfactory results in the reduction of GF.

Keywords: Cellulitis; Body Image; Electric Stimulation Therapy; Ultrasonic Therapy; Phonophoresis.
} 


\section{Introdução}

Desde que o conceito de saúde passou a ser definido como um estado de completo bemestar físico, mental e sociall, ${ }^{1}$, pode-se compreender que os distúrbios estéticos são uma ameaça à integridade emocional do indivíduo. Alguns desses distúrbios, de elevada incidência na população feminina, são agravados pela presença de fibro edema gelóide (FEG).

O FEG, conhecido popularmente como celulite, é uma afecção de origem multifatorial, causado por dietas inadequadas, tabagismo e efeitos hormonais, intensificados pelo o uso de anticoncepcionais, entre outros ${ }^{2,3}$. O local com FEG possui aumento no número e volume de adipócitos, bem como retenção de líquidos que comprimem os vasos, comprometendo a circulação sanguínea no local. Consequentemente, há rompimento de fibras de colágeno e elastina, que ocasiona deformações nos tecidos, hipotonia muscular e, em casos mais avançados, dor espontânea ou ao toque ${ }^{3,4,5}$. Embora alguns autores considerem a FEG inevitável e seu tratamento com caráter fútil ${ }^{6}$, as pessoas com esse problema apresentam sintomas físicos e alteração da imagem corporal, associados a problemas emocionais ${ }^{3}$.

Diversas terapias têm como objetivo o tratamento do FEG baseado na redução da gordura subcutânea. Porém, o desenvolvimento de métodos terapêuticos mais eficazes e que contemplem diferentes aspectos como a redução da fibrose, da flacidez muscular, do edema tecidual e o aumento da tonificação cutânea, além do acúmulo de gordura, ainda se faz necessário ${ }^{6,7}$. A Fisioterapia Dermatofuncional utiliza diversas técnicas aprimoradas para manter o padrão de beleza da sociedade atual e disponibiliza diferentes opções de tratamento para o FEG ${ }^{5,7}$.

Dentre eles, o ultrassom (US) terapêutico pode causar o rompimento de membranas e tecidos celulares por explorar a transformação de energia acústica (mecânica) em energia térmica $^{8}$. Dessa forma, a micromassagem induzida pelo US reduz a sintomatologia álgica e auxilia no rearranjo e extensibilidade das fibras colágenas. O US provoca alterações arquitetônicas na gordura subcutânea, interrompendo a estrutura do FEG e sua covinha decrescente ${ }^{3,4}$. Ainda, o US possibilita o aumento da circulação sanguínea, reduz o edema e auxilia no relaxamento muscular local ${ }^{3,6}$. De forma importante, o US facilita a veiculação de substâncias medicamentosas e aumento circulatório através da pele pela fonoforese (FN). Na FN, acrescenta-se ao gel condutor do US, produtos derivados de plantas ou drogas com princípios ativos que possam atuar no $\mathrm{FEG}^{3,4,9,10}$.

Outro recurso interessante nessa área é a eletroestimulação, através da aplicação de correntes excito motoras, como a corrente russa $(\mathrm{CR})^{11}$. O emprego da CR na melhora do aspecto da pele fundamenta-se pelo fortalecimento e/ou hipertrofia muscular, bem como a melhora/aumento da circulação sanguínea e linfática, auxiliando assim na melhora do aspecto trófico dos tecidos moles na região tratada ${ }^{3}$.

Portanto, a proposta deste estudo foi verificar o efeito do US e da FN, ambos associados à $\mathrm{CR}$, no tratamento da FEG em glúteos e coxas de mulheres jovens, averiguando sua distorção da imagem corporal e seu grau de satisfação com o tratamento proposto. Ainda, buscamos comparar o resultado desses recursos fisioterápicos sobre os sinais do FEG.

\section{Material e métodos}

\section{Caracterização da Ámostra}

Esse estudo clínico aberto, prospectivo e de intervenção terapêutica, apresenta os resultados das avaliações antes e depois dos tratamentos propostos e a comparação entre os resultados deles ao final das terapias. Esse estudo possui população amostral não aleatória, selecionada por conveniência, de acordo com a adesão das participantes. O cálculo da amostra foi realizado por número de medições dentro de um período de tratamento (junho de 2016 a junho de 2017). Nesse sentido, foi publicada em cartazes 
a possibilidade de tratamento para o FEG nos locais de estágio da Universidade Federal do Pampa (Unipampa) - postos de saúde e laboratórios. Embora tenhamos recebido muito retorno, apenas 23 voluntárias realizaram a avaliação inicial. No entanto, duas delas estavam realizando outros tratamentos estéticos e duas não indicaram tempo hábil para a realização da terapêutica. Desta forma, apenas 19 voluntárias iniciaram e terminaram os tratamentos propostos. As participantes eram do sexo feminino, foram recebidas por livre demanda, esclarecidas previamente sobre o estudo e assinaram um Termo de Consentimento Livre e Esclarecido (TCLE). O estudo foi aprovado pelo Comitê de Ética e Pesquisa da Unipampa, em consonância com a resolução 466/12, do Conselho Nacional de Saúde, sob parecer n. 1.759.472.

Para seleção das voluntárias foram traçados fatores de inclusão: possuir mais de 18 anos; exibir algum grau de FEG na região de coxas e/ ou glúteos; apresentar comportamento sedentário (sem realizar atividade física regular); no momento da pesquisa não estar realizando nenhuma outra intervenção para FEG; sem problemas hepáticos; sem alergias aos produtos utilizados na pesquisa e que assinaram o TCLE. Como fatores de exclusão: ter realizado cirurgia estética recentemente; estar fazendo uso de medicação para perda de peso; ser gestante; apresentar alterações de sensibilidade; ser portador de marca-passo cardíaco, prótese ou implante metálico no local de aplicação das técnicas (coxas e/ou glúteos); possuir doenças cardíacas e/ ou renais, circulatórias - como tromboflebite, alterações hormonais graves, infecção aguda; acometimento por lesão aberta no local de aplicação do tratamento; possuir áreas tratadas por radioterapia e tumores no local do tratamento.

\section{Avaliação}

Todas as voluntárias foram submetidas a uma avaliação e a uma reavaliação, realizadas por um examinador que não sabia sobre os tra- tamentos (cego). A coleta dos dados foi efetuada do seguinte modo:

1. Ficha de anamnese composta por: dados pessoais; histórico; critérios de inclusão e exclusão; tratamento estético anterior; antecedentes alérgicos; funcionamento intestinal; prática de exercício físico; tabagismo; alimentação; uso de medicamentos; portador de epilepsia; antecedentes oncológicos; disfunção hormonal; transtorno circulatório e/ou de cicatrização; propensão a queloides; doença dérmica; ciclo menstrual regular/ menopausa e método anticoncepcional.

2. Exame físico e dados antropométricos: cor da pele; trofismo e/ou flacidez muscular; adiposidade localizada e, dados antropométricos: massa (peso); altura; índice de massa corporal (IMC); perimetria; adipometria; verificado o grau de acometimento do FEG e registro fotográfico:

- Índice de massa corporal (IMC): foi definido após mensuração do peso e a medição da altura (balança camry), usando a fórmula: IMC=peso/(altura) ${ }^{2}$.

- Perimetria: a espinha ilíaca ântero-superior (EIAS) direita e esquerda foi usada como ponto fixo para a escolha dos locais de avaliação das medidas corporais. Com a voluntária em posição ortostática, foram realizadas demarcações a $15 \mathrm{~cm}$ (ponto 1), $20 \mathrm{~cm}$ (ponto 2) e $25 \mathrm{~cm}$ (ponto 3) abaixo da EIAS. As medidas foram realizadas com a fita métrica, na altura das três demarcações, bilateralmente em torno das coxas das voluntárias. Os dados foram expressos como a média das medidas nos três locais.

- Adipometria: em posição ortostática, foi realizado o pinçamento da dobra cutânea em membros inferiores, com o polegar e o dedo indicador. As medidas das dobras verticais foram obtidas com adipômetro (da marca Pró Fisiomed) em dois pontos: 1 (três centímetros acima da patela) e 2 (seis centímetros acima da patela). As medidas 
foram realizadas nas duas pernas do indivíduo, três vezes em cada ponto e utilizouse a média de todos os valores para cálculo estatístico.

- Grau de acometimento do FEG: a avaliação foi realizada mediante inspeção e palpação através dos testes de "casca de laranja" e "preensão" para identificar irregularidades e ondulações na superfície da pele ${ }^{3,10}$.

- Registro fotográfico: realizado em frente a uma parede de cor clara com a máquina fotográfica FUJIFILM S2000 posicionado a $65 \mathrm{~cm}$ de distância da voluntária e a $85 \mathrm{~cm}$ de altura. O registro foi realizado antes do início da primeira sessão e após o término da décima sessão de tratamento, com as voluntárias em posição ortostática e musculatura dos glúteos relaxada e, posteriormente, contraída, compondo um comparativo visual entre as fotos.

3. Body Shape Questionnaire (BSQ): o escore de insatisfação sobre a imagem corporal foi avaliado através da escala BSQ, composta por 34 itens, todos objetivos, respondidos em uma escala de cinco pontos (modelo Likert). Cada questão apresenta seis possibilidades de resposta: 1) Nunca; 2) Raramente; 3) Às vezes; 4) Frequentemente; 5) Muito Frequentemente e 6) Sempre. A distorção de imagem corporal (DIC) pode ser leve (70 a 90), moderada (90 a 110) ou intensa $(>110)$.

Além disso, foi aplicado um questionário de satisfação - apenas ao final das sessões (na reavaliação). Nele, foi realizada a pergunta "Qual sua opinião a respeito dos resultados do tratamento?", seguida de uma escala de 0 a 10 verificando o grau de satisfação pessoal quanto ao resultado em glúteos e coxas, separadamente.

\section{Protocolo de Tratamento}

Foram realizadas 10 sessões ( $3 x /$ semana), com CR associada ao US ou à FN aplicadas nos glúteos e na parte posterior das coxas. As participantes deitavam-se na maca em decúbito ventral, com os membros inferiores estendidos e relaxados. Neste momento era realizada uma esfoliação e higienização do local de tratamento com algodão e álcool 70\%. Após esse procedimento, foi realizada a aplicação da CR (aparelho Neurodyn dez canais - Ibramed@), utilizando-se 8 canais do aparelho (Figura 1). Os parâmetros da CR foram: frequência de $2500 \mathrm{~Hz}$ em bursts de $50 \mathrm{~Hz}$ (frequência modulada); recrutando teoricamente mais fibras brancas; ciclo de trabalho de $50 \%$; 3 segundos (s) de subida, 8 s de sustentação, $3 \mathrm{~s}$ de descida e $12 \mathrm{~s}$ de repouso, com duração total de 15 minutos. A intensidade da corrente era regulada conforme a tolerância de cada participante, verificando-se a contração da musculatura. Ao fim da terapia, os eletrodos foram retirados e a pele higienizada para aplicação do US ou FN. O Grupo 1 (G1) foi definido como as participantes que realizariam $\mathrm{US}+\mathrm{CR}$; e o Grupo 2 (G2) as voluntárias que seriam tratadas com FN + CR.

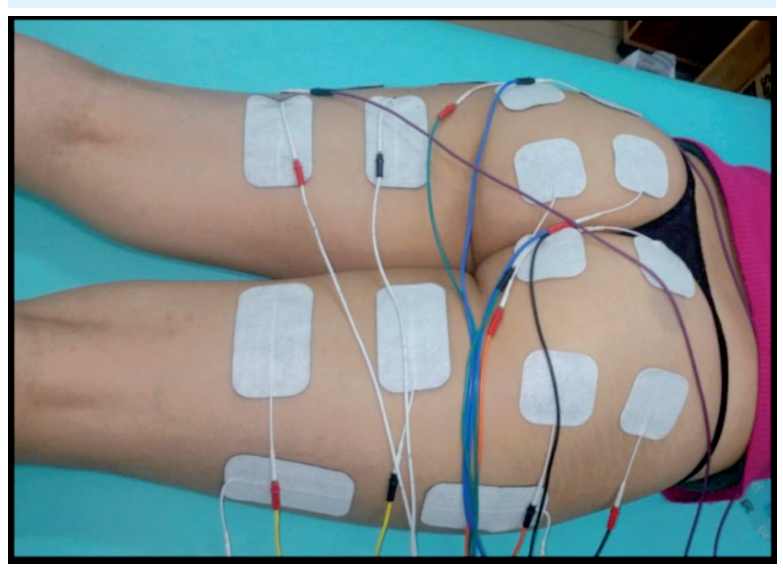

Figura l: Demonstração da posição dos eletrodos

Foram utilizados 8 canais, com dois eletrodos autoadesivos nos isquiostibiais $(9 \times 5 \mathrm{~cm})$, dois nos abdutores $(9 \times 5 \mathrm{~cm})$ e quatro em cada glúteo $(5 \times 5 \mathrm{~cm})$.

Fonte: Próprios autores.

Para a aplicação do US e da FN, foi utilizado o aparelho Sonopulse III (IBRAMED@). Na realização do US utilizou-se um gel condutor neutro de carbopol. Na realização da FN a com- 
posição do gel foi à base de extrato glicolítico de castanha da índia 3\%, extrato glicolítico de centella asiática $4 \%$, extrato glicolítico de cavalinha $3 \%$, cafeína $5 \%$ e ginkgo biloba $5 \%{ }^{12,13}$. O US, para ambos os tratamentos, foi aplicado na forma direta, no modo contínuo, com frequência de $3 \mathrm{Mhz}$ e intensidade de $1,0 \mathrm{w} / \mathrm{cm}^{2}$ e como tempo de aplicação utilizou-se Tempo = Área/ ERA $\left(E R A=3,5 \mathrm{~cm}^{2}\right)^{14}$. As áreas tratadas variaram de acordo com as necessidades de cada participante, sem determinar padronização da aplicação. Contudo, todas as voluntárias foram tratadas com tempos similares, não ultrapassando 20 minutos de aplicação. Ao término do tratamento com US ou FN, o gel foi removido, a pele higienizada e a voluntária era liberada.

O tratamento foi realizado por aproximadamente 45-50 minutos, incluindo também assepsia, colocação dos eletrodos e limpeza após tratamento. No último dia de realização do protocolo, após as 10 sessões de tratamento, a voluntária foi reavaliada conforme descrição anterior.

\section{Anólise estatística}

$\mathrm{Na}$ análise estatística os resultados foram analisados utilizando-se teste $t$ para dados paramétricos (perimetria, adipometria, IMC, grau de satisfação com o tratamento), sendo esses apresentados como média \pm desvio padrão (DP). O teste de Wilcoxon foi utilizado para análise de dados não paramétricos (Body Shape Questionnaire) e os resultados foram expressos como mediana \pm DP. Nesses casos, comparouse o antes e o depois da aplicação da terapia no mesmo grupo experimental ou os dois grupos experimentais entre si. A análise estatística foi realizada usando-se o Software GrafhPad (San Diego, CA, USA). O nível de significância em todos os casos foi considerado $p<0,05$. Para verificar o tamanho de efeito comparamos as médias e usamos o d de Cohen, verificando o grau ou dimensão em que o fenômeno está presente na população.

\section{Resultados}

As 19 voluntárias foram separadas em dois grupos: G1) US + CR (n=9) e G2) FN + CR (n=10). Ambos os tratamentos foram realizados na região de coxas e glúteos.

As participantes apresentavam idade média de $25 \pm 2,6$ anos, no G1 e de 23,5 \pm 3 anos, no G2. Quanto à ocupação, duas (2) voluntárias eram comerciárias, duas (2) eram professoras de ensino médio e superior, e quinze (15) eram estudantes de graduação. Sobre seus hábitos de vida, dezoito (18) mulheres referiram fazer uso de anticoncepcional (4-14 anos); seis (6) relataram uso de bebida alcoólica aos finais de semana. Em relação à ingestão de refrigerantes, oito (8) voluntárias referiram fazer uso diário dessas bebidas. Sobre a quantidade de água ingerida por dia, onze (11) consumiam abaixo de um litro por dia e o restante, entre um e dois litros de água por dia. Com relação à alimentação, doze (12) mulheres referiram ter uma alimentação gordurosa. Com relação à história familiar, 100\% relatou ter algum familiar com FEG. Duas voluntárias haviam realizado tratamento estético para FEG anteriormente (mais de 6 meses antes do atual estudo). Na reavaliação as voluntárias relataram que não modificaram seus hábitos de vida, ingestão de bebidas ou alimentação durante a realização do tratamento.

As mulheres apresentavam IMC médio de $24,8 \mathrm{~kg} / \mathrm{m}^{2}$ no grupo US + CR (G1) e $23,4 \mathrm{~kg} / \mathrm{m}^{2}$ no grupo FN + CR (G2). Ao final do tratamento, não houve diferença estatística quanto ao IMC ou peso das voluntárias.

Os resultados demonstram que as voluntárias do grupo US + CR tiveram redução da perimetria de coxas, enquanto no grupo $\mathrm{FN}+\mathrm{CR}$ não foi observado diferença estatística, comparando-se antes e depois (Tabela 1).

$\mathrm{Na}$ Tabela 1 ainda estão demonstrados os resultados da adipometria, onde se pode observar que ambos os grupos de tratamento apresentaram redução neste parâmetro. Pode-se verificar na Figura 2 A que houve redução significativa nas duas dobras cutâneas avaliadas 
em ambos os grupos de tratamento $(\mathrm{p}<0.05)$. No entanto, pode-se observar que houve uma maior porcentagem de redução das dobras cutâneas no grupo FN + CR comparado ao grupo US + CR, como observado na Figura 2B.

Tabela l: Comparação dos resultados antes e depois da perimetria e da adipometria em voluntórias para o tratamento da FEG

\begin{tabular}{ccc}
\hline \multicolumn{3}{c}{ Perimetria (EM CM $\left.{ }^{2}\right)$} \\
& US + CR & FN + CR \\
\hline Antes & $58.48 \pm 6.41$ & $56.59 \pm 5.69$ \\
Depois & $57.10 \pm 6.20$ & $56.26 \pm 5.83$ \\
$P$ & $<0.0001$ & 0.0513 \\
$d$ de Cohen & 0.0003145 & 0.057277 \\
Tamanho do efeito & 0.0001573 & 0.028627 \\
Redução & $1.38 \pm 2.13$ & $0.33 \pm 1.47$ \\
\hline
\end{tabular}

\begin{tabular}{ccc}
\multicolumn{3}{c}{ Adipometria (EM MM) } \\
& US + CR & FN + CR \\
\hline Antes & $34.38 \pm 7.25$ & $25.59 \pm 9.83$ \\
Depois & $31.41 \pm 7.06$ & $19.56 \pm 0.91$ \\
$P$ & 0.0002 & $<0.0001$ \\
$d$ de Cohen & 0.4150887 & 0.8640187 \\
Tamanho do efeito & 0.2032138 & 0.3965839 \\
Redução & $3.00 \pm 4.06$ & $6.03 \pm 6.26$ \\
\hline
\end{tabular}

Comparação dos resultados antes e depois da perimetria e da adipometria (dados das duas dobras cutâneas somadas), entre os tratamentos com ultrassom terapêutico associado à corrente russa (US $+\mathrm{CR}$ ) e fonoforese associada à corrente russa (FN + CR). Colunas "antes", "depois" e "redução" demostram valores de média \pm desvio padrão da média. Fonte: Próprios autores.

Quanto ao questionário sobre a imagem corporal (BSQ) podemos perceber que ambos os tratamentos reduziram a distorção da imagem corporal (DIC) das voluntárias $(\mathrm{p}<0.05$ e $\mathrm{p}<0.01$ para G1 e G2, respectivamente), como observado na Tabela 2. Verificamos também que, comparando os resultados finais dos grupos experimentais, nota-se que os níveis de DIC tiveram redução mais significativa no grupo $\mathrm{FN}+\mathrm{CR}$ do que no grupo US + CR (Tabela 2).

Para melhor visualização do resultado do questionário de satisfação com a imagem corpo-

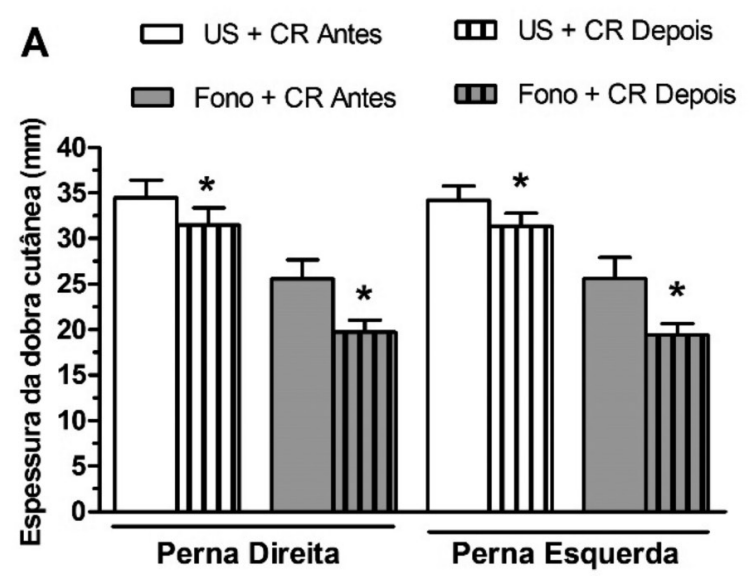

B

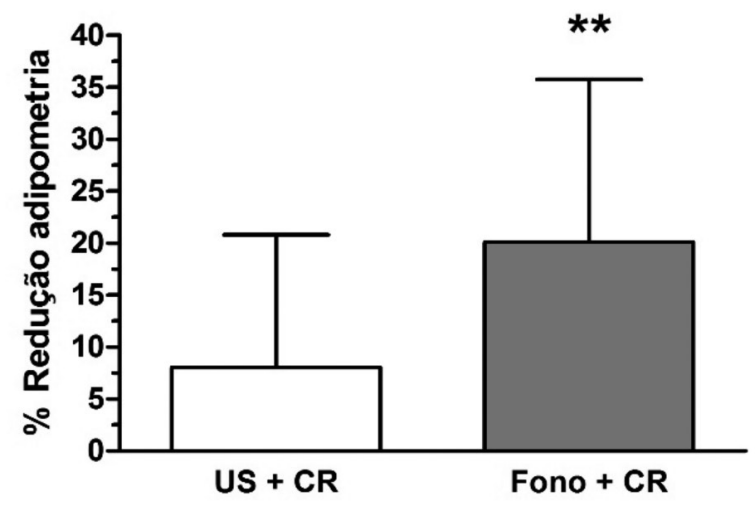

Figura 2: Avaliação das dobras cutâneas dos indivíduos com FEG antes e após o tratamento com US + CR ou FN + CR.

(A) representa as duas dobras cutâneas avaliadas em cada membro inferior. (B) representa a porcentagem de redução da adipometria - consideramos 100\% o valor inicial e quanto foi reduzido individualmente. Cada coluna representa a média \pm DP da espessura da dobra cutânea de 9 ou 10 indivíduos. Os asteris$\cos (*)$ demonstram os níveis de significância quando comparados com o valor antes do tratamento (antes), ${ }^{*} p<0.05$ e ${ }^{* *} p<0.01$.

Fonte: Próprios autores.

ral - BSQ - os dados individuais das participantes com a DIC, descritos por elas mesmas, estão disponibilizados na Tabela 3.

Verificou-se ainda, através do teste "casca de laranja" e do teste de "preensão", que houve redução no grau da FEG em duas voluntárias do tratamento com US + CR e apenas uma voluntária do tratamento com FN + CR. Porém, foi observada melhora da aparência da pele e do tônus 
Tabela 2: Valores coletados na aplicação do questionário sobre a distorção com a imagem corporal (DIC)

\begin{tabular}{|c|c|c|c|}
\hline \multicolumn{3}{|c|}{ Body Shape Questionnaire (BSQ) } & \multirow[b]{2}{*}{$\Delta$} \\
\hline & US + CR & $\mathrm{FN}+\mathrm{CR}$ & \\
\hline Antes & $117.00 \pm 29.05$ & $98.00 \pm 30.31$ & $25.59 \pm 9.83$ \\
\hline Depois & $88.00 \pm 25.38$ & $88.00 \pm 32.06$ & $19.56 \pm 0.91$ \\
\hline$P$ & 0.0207 & 0.0059 & $<0.0001$ \\
\hline$d$ de Cohen & 0.7995808 & 0.4423464 & 0.8640187 \\
\hline Tamanho do efeito & 0.3712229 & 0.2159543 & 0.3965839 \\
\hline Redução & $21.78 \pm 13.80$ & $13.80 \pm 8.80$ & $6.03 \pm 6.26$ \\
\hline
\end{tabular}

DIC antes e depois do tratamento com Ultrassom Terapêutico associado à corrente russa (US + CR) e tratamento com fonoforese associado à corrente russa (Fono + CR). As linhas "antes", "depois" e "redução" demostram valores de mediana \pm desvio padrão. Fonte: Próprios autores.

Tabela 3: Resultado bruto do questionário de satisfação com a imagem corporal - BSQ - antes e depois dos tratamentos para FEG

\begin{tabular}{|c|c|c|c|c|}
\hline Voluntária & Antes & $\begin{array}{c}\text { Distorção } \\
\text { da imagem } \\
\text { corporal }\end{array}$ & Depois & $\begin{array}{c}\text { Distorção } \\
\text { da imagem } \\
\text { corporal }\end{array}$ \\
\hline \multicolumn{5}{|c|}{ Tratamento com US + CR } \\
\hline 1 & 82 & Leve & 82 & Leve \\
\hline 2 & 101 & Moderada & 76 & Leve \\
\hline 3 & 117 & Intensa & 102 & Moderada \\
\hline 4 & 166 & Intensa & 126 & Intensa \\
\hline 5 & 142 & Intensa & 86 & Leve \\
\hline 6 & 108 & Moderada & 58 & Não possui \\
\hline 7 & 129 & Intensa & 120 & Intensa \\
\hline 8 & 143 & Intensa & 134 & Intensa \\
\hline 9 & 80 & Leve & 88 & Leve \\
\hline \multicolumn{5}{|c|}{ Tratamento com FN + CR } \\
\hline 1 & 86 & Leve & 74 & Leve \\
\hline 2 & 134 & Intensa & 120 & Intensa \\
\hline 3 & 117 & Intensa & 102 & Moderada \\
\hline 4 & 110 & Moderada & 104 & Moderada \\
\hline 5 & 86 & Leve & 56 & Não possui \\
\hline 6 & 54 & Não possui & 50 & Não possui \\
\hline 7 & 71 & Leve & 45 & Não possui \\
\hline 8 & 125 & Intensa & 109 & Moderada \\
\hline 9 & 46 & Não possui & 34 & Não possui \\
\hline 10 & 113 & Intensa & 110 & Moderada \\
\hline
\end{tabular}

Fonte: Próprios autores.

muscular em todas as participantes, de ambos os grupos experimentais. A melhora no aspecto da pele e a redução da FEG pode ser evidenciada através das fotos obtidas antes e após a rea- lização dos tratamentos (Figura 3). Essas imagens foram mostradas às voluntárias proporcionando um retorno visual sobre o resultado dos tratamentos a que elas foram submetidas.

Além disto, o estudo avaliou o índice de satisfação das mulheres com os resultados finais dos tratamentos recebidos. O grupo US + CR apresentou média e desvio padrão de $8,3 \pm 1,2$, enquanto o grupo $F N$ + CR apresentou a média e desvio padrão de $8,9 \pm 0,97$. Portanto, ambos os tratamentos foram satisfatórios para as participantes e não foi constatada diferença estatística entre os grupos na análise dessa variável.

\section{Discussão}

Poucas pesquisas científicas comprovam a efetividade das técnicas de fisioterapia na redução da FEG, ocasionando maior dificuldade em determinar evidências sobre o assunto ${ }^{15}$. A avaliação da eficácia do tratamento geralmente é subjetiva $^{16}$ e a avaliação da FEG em si é bastante questionada. Alguns autores relatam que cabe ao profissional considerar os fatores desencadeantes, pois são fundamentais para uma boa anamnese e abordagem clínica do $\mathrm{FEG}^{17}$. Por isso, existe a necessidade de uma combinação de mudança nos hábitos de vida que deve estar aliado aos tratamentos estéticos, uma vez que técnicas isoladas não são capazes de agir em todas as alterações promovidas pelo $\mathrm{FEG}^{18}$. Neste trabalho, o tratamento com US ou com $\mathrm{FN}$ associados a $\mathrm{CR}$ reduziram as medidas corporais e aspecto do FEG, bem como melhoram a própria imagem corporal das voluntárias. 


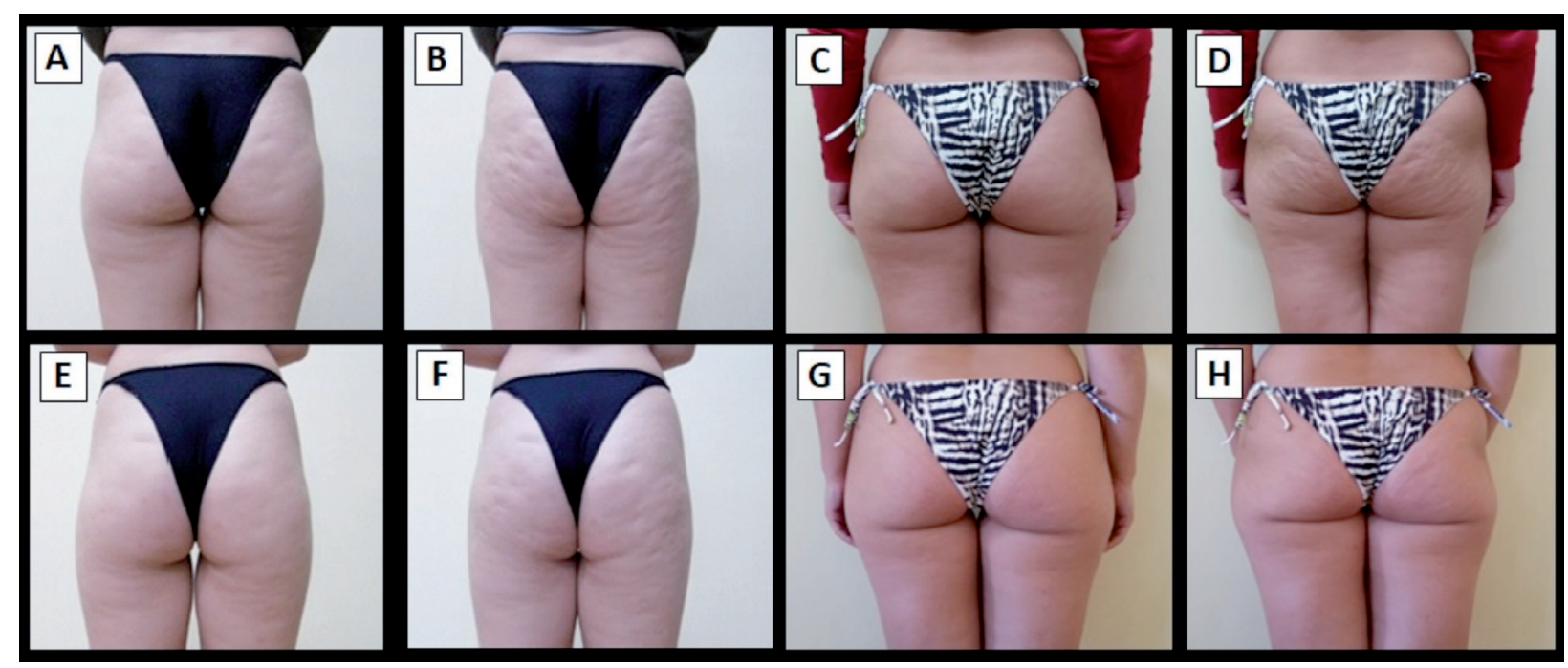

Figura 3: Imagens fotográficas obtidas antes e após tratamento com US ou FN associado ò CR em glúteos e coxas de voluntórias com FEG

As imagens em A, B, E e F representam uma voluntária tratada com US + CR. As imagens em $C, D, G$ e H representam uma voluntária tratada com $\mathrm{FN}+\mathrm{CR}$. (A e C) representam glúteos e coxas sem contração muscular e ( $B$ e D) glúteos e coxas com contração muscular - antes dos tratamentos para FEG. (E e G) representam glúteos e coxas sem contração muscular e ( $\mathrm{F}$ e H) glúteos e coxas com contração muscular - após 10 sessões para tratamento da FEG.

Fonte: Próprios autores.

O US é um dos mais requisitados pelos profissionais, porque seus efeitos no tecido corporal que auxiliam no tratamento do $\mathrm{FEG}^{8,9}$. O US é um método terapêutico que possui efeitos mecânicos como micromassagem, que pode propiciar a diminuição da dor, rearranjo e extensibilidade das fibras colágenas e melhora das propriedades mecânicas do tecido. Sua ação também promove efeito anti-inflamatório e aumento da circulação local ${ }^{3,8}$. Isso é importante porque no FEG ocorre alteração da matriz intersticial, estase microcirculatória e hipertrofia dos adipócitos, estimulando a retenção de líquidos e aumento de volume da célula - causando compressão dos vasos e o rompimento das fibras de colágeno e elastina ${ }^{3}$. De fato, o US, associado ou não à fonoforese, tem se mostrado uma forma eficaz na redução de problemas como o FEG e melhora da satisfação dos usuários dessa terapia ${ }^{14,19,20}$. No presente estudo, utilizou-se o US associado à CR e todas as participantes apresentaram melhora no aspecto da pele e redução da FEG, sendo confirmado por meio da análise subjetiva das fotografias, pela adipometria e pela inspeção visual nas mulheres, realizada após 10 sessões de tratamento.

Ainda, sabemos que a FN descreve a habilidade do US em incrementar a penetração de agentes farmacologicamente ativos através da pele. Ela apresenta várias vantagens como: ação localizada do princípio ativo; ausência de efeitos colaterais de ação sistêmica e o somatório dos efeitos do US associados aos efeitos das propriedades químicas dos princípios ativos ${ }^{21}$. A FN promove a neovascularização com consequente aumento da circulação, rearranjo e aumento da extensibilidade das fibras de colágeno e melhora das propriedades mecânicas do tecido ${ }^{22}$. Nesse trabalho, um dos grupos de mulheres foi tratado com FN + CR, onde observamos que as participantes também apresentaram melhora no aspecto da pele e redução da FEG, bem como da adipometria.

Por ser um distúrbio multifatorial, o tratamento do FEG deve contemplar diferentes aspectos e se direcionar a tratar a fibrose, o acúmulo de gordura, a tonificação cutânea e o edema teci- 
dual, bem como a flacidez muscular ${ }^{7}$. No entanto, existe uma grande escassez de trabalhos que mostrem o efeito de tratamentos que reduzem a flacidez, como correntes excitomotoras, no tratamento do FEG. A CR é uma corrente de média frequência, que estimula neurônios motores, induz a contração muscular através da despolarização das membranas e auxilia no aumento do trofismo e fortalecimento muscular, reduzindo a aparência da flacidez muscular, bem como a melhora da circulação linfática e sanguínea. Porém, é importante ressaltar que quanto maior a intensidade da corrente que o paciente suportar, maior será o montante de energia e maiores serão as unidades motoras recrutadas ${ }^{3,21,23}$. A associação do FEG com a flacidez muscular é um fato importante e decorrente da vida sedentária ou da prática de exercícios leves, que não são suficientes para obter um aumento e manutenção da massa muscular ${ }^{3}$.

Verificamos ainda, que apenas o grupo tratado com US + CR apresentou redução na perimetria da coxa. No entanto, esse resultado sozinho não representa significância clínica, tendo em vista que a CR favorece o aumento do trofismo muscular, o que poderia contribuir para a manutenção da perimetria mesmo que houvesse redução da gordura local. Observamos também que ambos os tratamentos reduziram a adipometria da coxa, porém, o tratamento com FN apresentou uma maior porcentagem de redução desse parâmetro. Sabe-se que o US tem demonstrado efeito sobre o FEG ${ }^{14,24,25}$, especialmente quando associado a outras terapias, como a endermoterapia ${ }^{26}$, ou associado a um gel com princípios ativos ${ }^{27}$. Ainda, o uso do US com gel anticelulíticos (FN) mostrou-se superior em eficácia ao US com gel comum, estimulando e acelerando significativamente a permeação de fármacos, levando assim à diminuição mais efetiva do $\mathrm{FEG}^{28}$. Os resultados do presente estudo validam a eficácia da formulação integral utilizada no tratamento com $\mathrm{FN}$, mas não fornecem informações sobre o desempenho de ingredientes individuais dessa formulação, uma limitação indicada por outros autores ${ }^{10}$.
O retorno visual das fotografias foi importante para que as mulheres pudessem verificar o resultado final do tratamento, embora, nos dois tipos de tratamentos, muitas delas já relatassem a melhora visual e no aspecto da própria pele antes mesmo do término das sessões. Essa demonstração de cuidado e visualização do próprio corpo é um importante fator de averiguação da imagem corporal. Os cuidados com o corpo tornam-se uma preocupação constante na vida das pessoas e a presença de FEG influência significativamente no bem-estar das mulheres, pois a imagem corporal atualmente é de fundamental importância para se ter uma boa autoestima ${ }^{7,8}$. Cerca de $50 \%$ da população refere algum tipo de insatisfação com a sua própria aparência e parte desse grupo procura algum tipo de procedimento para a correção dos traços considerados indesejáveis ${ }^{28}$. No trabalho atual, os dois grupos de tratamentos reduziram a distorção da imagem corporal das mulheres com FEG, que ficaram satisfeitas com os resultados obtidos. Para a mulher a melhora da própria imagem corporal torna-se importante porque a imagem da mulher e do feminino está associada à beleza, havendo cada vez menos tolerância para os desvios nos padrões estéticos socialmente estabelecidos ${ }^{29}$.

Ainda, as participantes do estudo ficaram satisfeitas com os resultados obtidos após a terapia. Realmente, estudos usando US isoladamente $^{14,25}$ ou com gel com princípios ativos ${ }^{26}$ demonstram que os indivíduos relatam aumento da satisfação com os resultados da terapia usada no tratamento da FEG. Aparentemente, o maior índice de satisfação do uso do US na FEG é em relação ao resultado obtido no aspecto geral do glúteo ${ }^{24}$. Embora no presente estudo as duas técnicas não tenham resultados diferentes entre si, outras pesquisas demonstram que a FN apresenta resultados mais satisfatórios do que o US isoladamente ${ }^{27}$.

Deve-se ressaltar que apesar dos resultados promissores da pesquisa atual, alguns autores demonstram pouca eficácia com uso do US e da FN no tratamento da FEG ${ }^{7}$. Ademais, infelizmente nesta pesquisa não foi possível o 
incremento de outros grupos experimentais que possibilitariam identificar os efeitos isolados de cada tratamento. De qualquer forma, é necessário destacar ainda que, além dos tratamentos utilizados aqui, alguns cuidados como alimentação saudável e atividade física, podem auxiliar ainda mais na redução da $\mathrm{FEG}^{3}$.

\section{Conclusão}

A partir dos resultados obtidos no estudo, conclui-se que ambas as terapêuticas apresentam resultado na redução dos graus de FEG em mulheres jovens, entretanto, a fonoforese associada à corrente russa, mostra superioridade na eficácia em reduzir os graus de FEG, além de melhorar a percepção sobre a satisfação com a imagem corporal das voluntárias da pesquisa. Este estudo propôs um comparativo entre técnicas e elege a aplicação de fonoforese associada à corrente russa como melhor opção de tratamento nesta situação. Porém, existem outras técnicas que podem ser empregadas na redução dos graus de FEG, salientando a necessidade de mais estudos comparativos que permitam comprovação da eficácia dos tratamentos e suas associações.

\section{Referências}

1. Constituição da Organização Mundial da Saúde em 1946. Disponível em: <http://www. direitoshumanos.usp.br/index.php/OMSOrganiza\%C3\%A7\%C3\%A3oMundial-daSa\%C3\%BAde/constituicao-daorganizacao-mundialda-saudeomswho.html>. Acesso em: 20. Maio.2018.

2. Draelus ZD, Marenus KD. Celulite, Etiology and Purported Treatment. Dermatol Surg. 1997;23:11771181

3. Guirro E, Guirro R. Fisioterapia Dermato Funcional. 3 Ed. São Paulo: Manole, 2007.

4. Moravvej H, Akbari Z, Mohammadian S, Razzaghi $Z$. Focused ultrasound lipolysis in the treatment of abdominal cellulite: an openlabel study. J Lasers Med Sci 2015;6:102-105.
5. Khan MH, Victor F, Rao B, Sadick NS. Treatment of cellulite: part I. Pathophysiology. J Am Acad Dermatol. 2010;62:361-370.

6. Nürnberger F, Müller G. So-called cellulite: an invented disease. J Dermatol Surg Oncol. 1978;4:221-229.

7. Angelino BS. Fibroedema geloide subcutâneo: qué conocemos de esta entidad clínica? Folia Dermatol Peru. 2003;14:38-42.

8. Juhász M, Korta D, Mesinkovska NA. A Review of the Use of Ultrasound for Skin Tightening, Body Contouring, and Cellulite Reduction in Dermatology. Dermatol Surg. 2018;44:949-963.

9. Ferreira LL, Fernandes C, Cavenaghi S. Fisioterapia no Fibroedema Geloide: Análise de Periódicos Nacionais. Revista de Atenção à Saúde. 2014;12:57-63.

10. Dupont E, Journet M, Oula ML, Gomez J, Léveillé C, Loing E, et al. An integral topical gel for cellulite reduction: results from a double-blind, randomized, placebo-controlled evaluation of efficacy. Clin Cosmet Investig Dermatol. 2014;7:73-88.

11. Pereira MFL. Eletroterapia: no tratamento Estético. 1. Ed. São Paulo: Difusão, 2014.

12. Ramalho TA, Curvelo S. Substâncias cosmetologicamente ativas caracterização, indicação, eficácia e segurança: cafeína. Ver. Lusófona de Ciências e Tecnologias da Saúde. 2006;3:183-190.

13. Lopes. E, Piacentini E, Santos P, Waltrick T, Thives RC, Santos D, et al. Análise comparativa do tratamento com Manthus ao FEG grau III utilizando gel comum ou gel com princípios ativos em mulheres de 27 à 42 anos de idade. Revista Científica Jopef - Editora Korppus. 2013;2:61-72.

14. Machado GC, Vieira RB, Oliveira NML, Lopes CR. Análise dos Efeitos do Ultrassom Terapêutico e da Eletrolipoforese nas Alterações Decorrentes do Fibroedema Geloide. Fisioterapia em Movimento. 2011;24:471-479.

15. Ottawa P. Evidence Based Clinical Practice Guidelines for Eletrotherapy and Thermotherapy Interventions in the Management of Rheumatoid Arthritis in Adults. Phys Ther. 2004;84:1016-1143.

16. Wanner M, Avram M. An evidence-based assessment of treatments for cellulite. J Drugs Dermatology. 2008;7:341-345.

17. Meyer PF, Lisboa FL, Alves MCR, Avelino MB. Desenvolvimento e aplicação de um protocolo de avaliação fisioterapêutica em pacientes com fibro edema gelóide. Fisioterapia em Movimento. 2005;18:75-83 
18. Manuskiatti W, Wachirakaphan C, Lektrakul N, Varothai S. Circumference reduction and cellulite treatment with a TriPollar radiofrequency device: a pilot study. J Eur Acad Dermatology Venereology. 2009;23:820-827.

19. de Almeida AF, Brandão DSM, Silva JC, Oliveira RGCQ, Araújo RC, Pitangui ACR. Avaliação do efeito da drenagem linfática manual e do ultrassom no fibroedema geloide. RBCS. 2011;9:31-37.

20. Fonseca NH, Moura WEM, Cardoso SBA, de Campos JC, Monteiro NA, França JS. A aplicabilidade do ultra-som de $3 \mathrm{MHZ}$ associado a fonoforese no tratamento do Fibro edema gelóide (FEG) na região glútea. Acta Biomedica Brasiliensia. 2013:4:106-113

21. Borges FS. Dermato-Funcional: Modalidades Terapêuticas nas Disfunções Estéticas. 2 Ed. São Paulo: Phorte, 2010.

22. Conti BZ, Pereira TD. Ultra-som terapêutico na redução da lipodistrofia ginóide. Fisio\&terapia. 2003;7:11-14.

23. Low J, Reed A. Eletroterapia Explicada: Princípios e Práticas. 3 Ed. São Paulo: Manole, 2001.

24. Cappellazzo R, Batista C, Marcelino DA, Nonino F, Machado MC, Yamazaki ALS. IX EPCC - Encontro Internacional de Produção Científica UniCesumar; 3-6 de novembro 2015; Maringá, PR, Brasil.

Maringá, 2015.
25. Almeida TP, Kilian T, Moreira JAR. Comparação entre a endermoterapia e o ultrassom no tratamento do fibro edema geloide. Revista Científica FHO|UNIARARAS. 2015;3:73-83

26. Felipe ODP, Moura WEM, Cardoso SBA, Campos JC, Monteiro AN, França JS. Aplicação da Fonoforese no Tratamento do Fibro Edema Gelóide na Região Abdominal. Acta Biomedica Brasiliensia. 2014;5:110-117

27. Waltrick T, Schüler E, Santos P, Waltrick JB, Santos D. Análise da eficiência do ultrassom terapêutico contínuo utilizando gel comum e gel com princípio ativo no tratamento do Fibro Edema Geloide grau II. Revista Inspirar. 2011;3:3-9

28. Hexsel D, Siega C, Schilling-Souza J, Porto MD, Rodrigues TC. A Comparative Study of the Anatomy of Adipose Tissue in Areas with and Without Raised Lesions of Cellulite Using Magnetic Resonance Imaging. Dermatologic Surgery. 2013;39:1877-1886.

29. Novaes JV, Vilhena J. De cinderela à moura torta: sobre a relação mulher, beleza e feiúra. Revista Interações. 2003;8:9-36. 